\title{
Editorial
}

\section{Functional Polymeric Materials Based on Cellulose}

\author{
Haisong Qi, ${ }^{1}$ Ang Lu, ${ }^{2}$ Qingbin Zheng, ${ }^{1}$ and Quanling Yang ${ }^{3}$ \\ ${ }^{1}$ Leibniz-Institut für Polymerforschung Dresden e.V., 01069 Dresden, Germany \\ ${ }^{2}$ College of Chemistry and Molecular Sciences, Wuhan University, Wuhan 430072, China \\ ${ }^{3}$ The University of Tokyo, Tokyo, Japan \\ Correspondence should be addressed to Haisong Qi; qi@ipfdd.de
}

Received 4 August 2016; Accepted 4 August 2016

Copyright (C) 2016 Haisong Qi et al. This is an open access article distributed under the Creative Commons Attribution License, which permits unrestricted use, distribution, and reproduction in any medium, provided the original work is properly cited.

Nowadays, functional materials based on renewable bioresources and environmentally friendly processes have attracted considerable attention. Cellulose is the most common organic polymer on earth, representing about $1.5 \times$ $10^{12}$ tons of the total annual biomass production, and is considered an almost inexhaustible source of raw material. Cellulose has fascinating structure and many attractive properties such as renewability, biodegradability, biocompatibility, and broad chemical-modifying capacity. It can be converted into various cellulose-based materials (such as fibers, films, membranes, composites, and biomedical materials) and has been used for more than one hundred years by man. Recently, the developments of new and "green" solvents for cellulose have provided novel platforms to prepare various cellulosebased materials through more efficient and environmentally friendly processes. New frontiers such as nanocellulose also offer great opportunities in the field of advanced and functional materials. In addition, advancements in nanomaterials and technologies are delivering rapidly new functional cellulose-based materials containing nanoparticles over a broad range of applications. The articles in this special issue cover the recent and significant research activities about cellulose-based functional materials.

Cellulose can be obtained from many natural resources, for example, wood, plant, bacteria, and algae. Due to their unique mechanical properties, the extracted cellulose-based natural fiber or nanocellulose can be used as fillers to enhance the properties of composite material. For example, cellulose fillers have the potential of enhancing the composite properties of plastics formed using acrylonitrile-butadiene-styrene (ABS), as well as increasing its biodegradation by organisms and recyclable nature. In this special issue, $\mathrm{K}$. Crews et al. in the paper titled "Influence of Cellulose on the Mechanical and Thermal Stability of ABS Plastic Composites" deal with the preparation and thermal stability of cellulose/ABS composites. They found that polymer dissolution was a useful technique for the improvement of cellulose-filled ABS composites. Y. Gao et al. in their paper titled "Preparation and Application of Cationic Modified Cellulose Fibrils as a Papermaking Additive" present modified cellulose fibrils by reacting the cellulose fibrils with 2,3-epoxypropyltrimethylammonium chloride (EPTMAC). After cationization, surface charge density and zeta potential of the cellulose fibrils reversed, while the surface morphology has no obvious change. The tear index of paper sheets was improved by addition of CMCF. In their paper titled "Long-Chain Alkylimidazolium Ionic Liquid Functionalization of Cellulose Nanofibers and Their Embedding in HDPE Matrix" C. Croitoru et al. report a new method for cellulose nanofibers functionalization, by treatment of the material at temperatures range of $25-35^{\circ} \mathrm{C}$ with electron-beam irradiated 1-hexyl-3-methylimidazolium chloride ionic liquid. The functionalized cellulose nanofibers present lower water vapor relative uptakes than the original ones. In addition, the grafting moieties also improved adhesion of the cellulose nanofibers to HDPE, thus contributing to obtaining polymer matrix composites with improved properties.

In the paper "Mechanical Properties of Oil Palm Shell Composites" of this issue, J. Sahari et al. find that oil palm shell (OPS) can improve the mechanical properties of the composites. Due to the hydroxyl groups on the fibers, the water absorption and moisture content of composites 
increased with increasing of OPS content. In the paper "Schizophyllum commune Lipase Production on Pretreated Sugarcane Bagasse and Its Effectiveness" of this issue, Schizophyllum commune UTARA1 was used for lipase production under solid state fermentation (SSF) of sugarcane bagasse (SB) impregnated with used cooking oil medium. Y. C. Kam et al. discussed its effectiveness in detail.

As well known, cellulose can be chemically modified to yield cellulose derivatives. In the paper "Homogeneous Esterification of Cellulose in the Mixture N-Butylpyridinium Chloride/Dimethylsulfoxide" of this issue, L. El Hamdaoui et al. deal with the homogeneous acylation of cellulose with pnitrobenzoyl chloride in a reaction medium composed of a mixture of 1-butylpyridinium chloride and dimethylsulfoxide (BPyCl/DMSO), in the presence of different bases and under mild conditions. It was shown that cellulose esters with different DS can be obtained by varying the reaction time in a completely homogeneous synthesis. The products can dissolve in some organic solvents such as DMSO, DMAc, and $\mathrm{DMF}$, depending on their DS values.

We hope that readers will benefit for their own knowledge and research from this special issue.

\section{Disclosure}

The current address for Quanling Yang is School of Materials Science and Engineering, Wuhan University of Technology, Wuhan 430070, China.

\section{Acknowledgments}

We thank all the authors for their valuable contributions and the reviewers for their great support to this special issue about cellulose.

Haisong Qi Ang Lu Qingbin Zheng Quanling Yang 

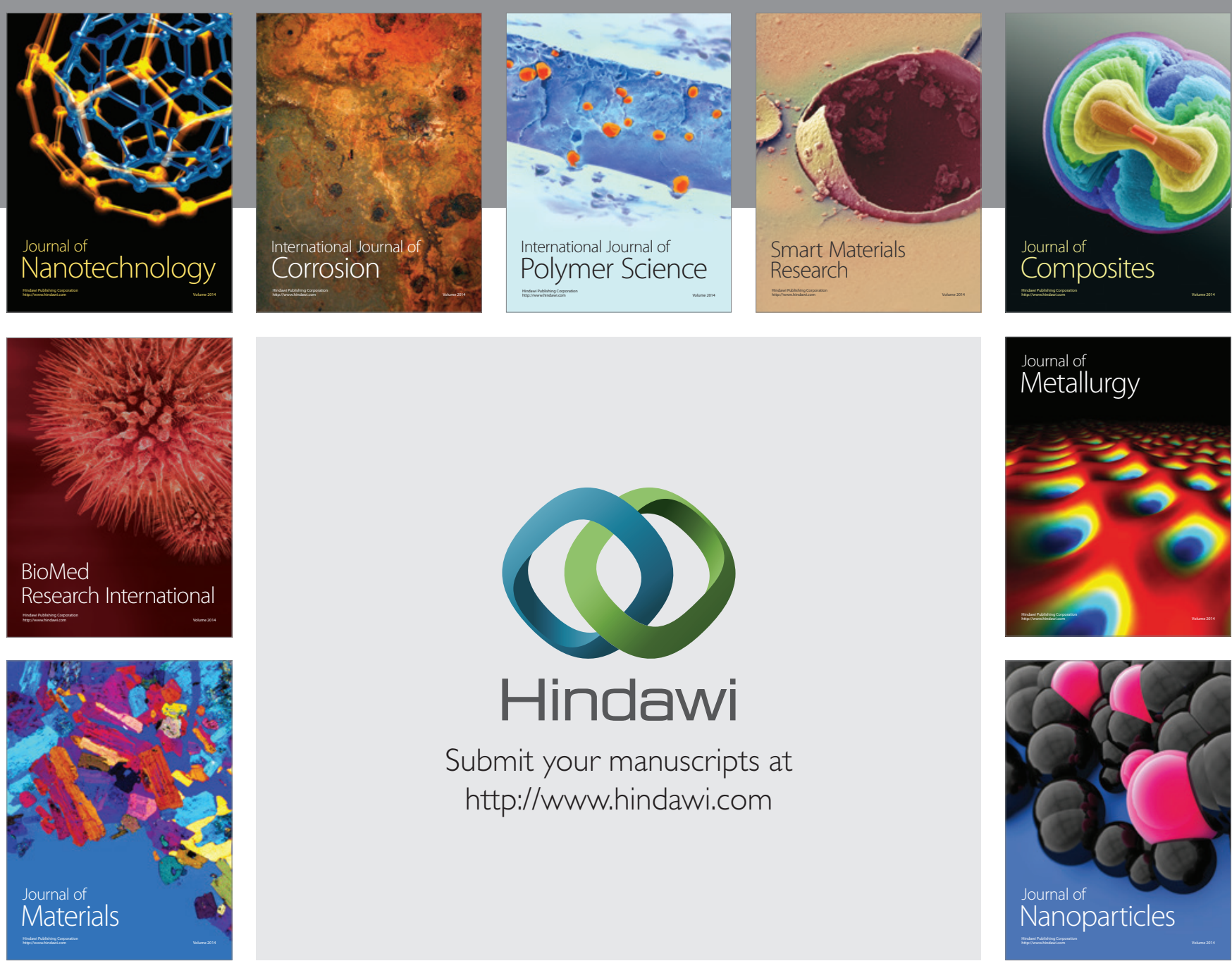

\section{Hindawi}

Submit your manuscripts at

http://www.hindawi.com

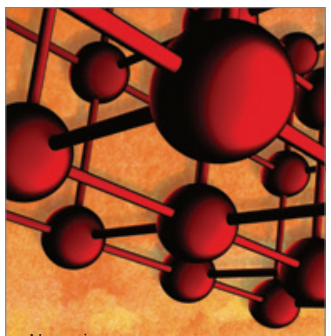

Materials Science and Engineering
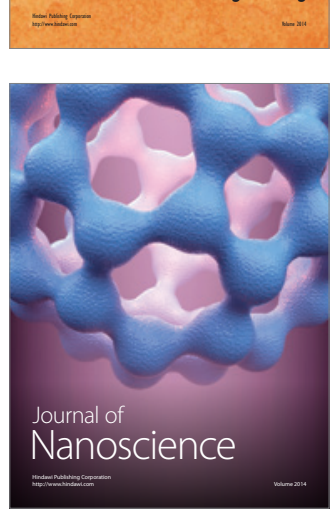
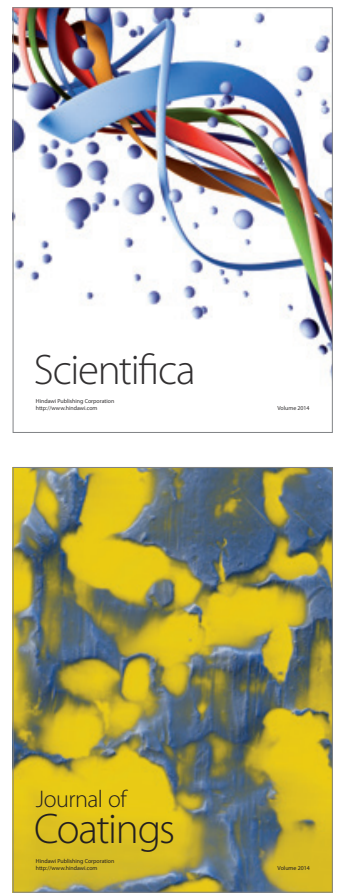
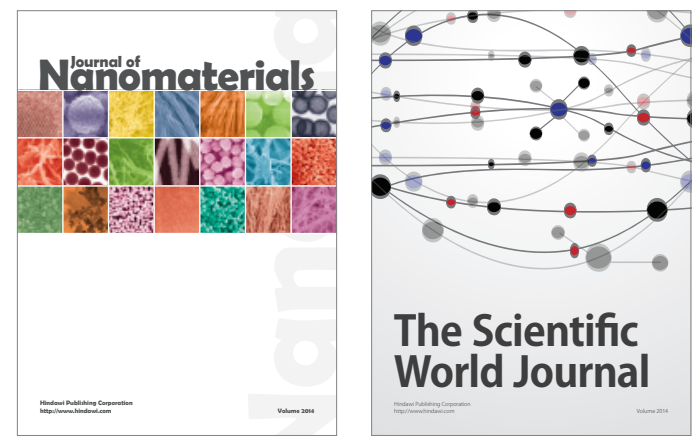

The Scientific World Journal
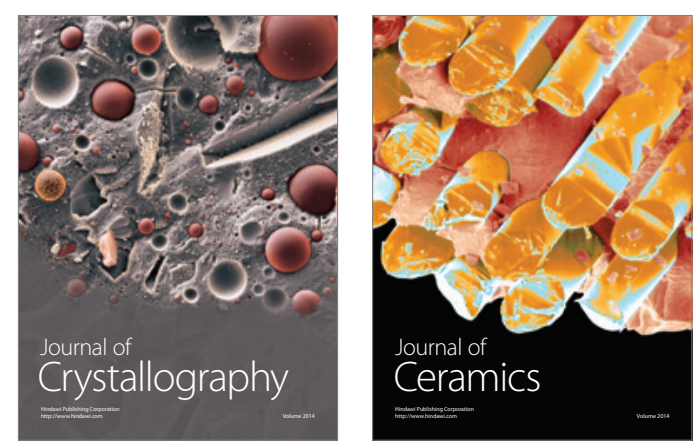
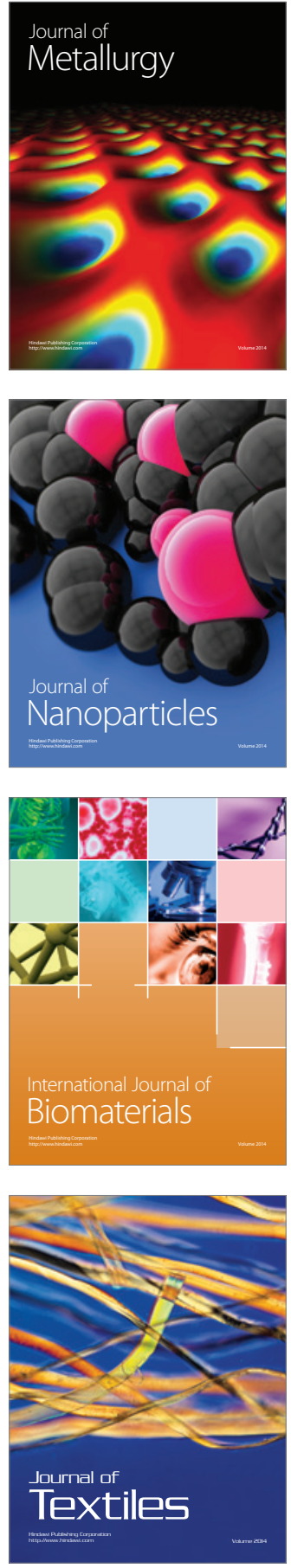